\title{
REMARQUES SUR LES CONSTRUCTIONS CAUSATIVES DU TYPE FAIRE + INFINITIF DANS LES LANGUES ROMANES ET QUELQUES AUTRES
}

\author{
MAREK GAWEŁKO \\ ul. Włoska $19 / 20$ \\ 30-638 Kraków \\ Pologne
}

Résumé : L'objectif de l'article est double : $1^{\circ}$ établissement de la vitalité des constructions causatives dans huit langues dont deux (l'anglais et le français) sont hautement analytiques, une (le polonais) hautement synthétique et cinq qui montrent une tendance analytique modérée, et $2^{\circ}$ explication typologique des différences observées.

La règle générale dit que la vitalité des constructions causatives est en rapport avec le degré d'analytisme de la langue où elles sont utilisées : elles sont les plus fortes en français et inexistantes en polonais. L'anglais fait exception à la règle: les constructions causatives y sont relativement rares, ce qui est en rapport avec la faiblesse relative de l'infinitif, concurrencé par les formes en -ing.

Mots clés : causatif, vitalité, explication, synthétique, analytique

\section{Remarques générales}

Les formes linguistiques porteuses d'une valeur causative ont été établies pour différentes langues du monde, y compris les langues romanes (cf. par ex. Shibatani 1976b; Givón-Young 2002; Shibatani 2002b; Iliescu 1995; Moeschler 2003, etc.). Les formes causatives les plus importantes sont: le causatif lexical (ex. tuer), le causatif morphologique (ex. fertiliser), le causatif syntaxique, par ex. du type faire + inf(initif). On indique aussi d'autres formes: participe, gérondif, constructions telles que préposition + inf, préposition + substantif, etc. (cf. Mori 1980, 30, note 13). Shibatani et Pardeshi $(2002,109)$ signalent des procédés tels que réduplication et 
apophonie. Il y a sans doute un rapport entre l'importance accordée aux types lexical, morphologique et syntaxique et le caractère typologique des langues où ils apparaissent.

Le causatif lexical est le moyen le plus fréquent d'exprimer la causalité. Cependant, il pose problème pour le linguiste vu que seul un critère sémantique permet de le distinguer du verbe non causatif. De plus, le caractère causatif du verbe devrait être établi dans le contexte où il est utilisé. Ainsi open n'est causatif que dans la deuxième phrase: The door opened. John opened the door (cf. Shibatani 1976a, 3). Il en va de même du verbe français aimer, qui n'est pas causatif. Cependant il le devient dans certains contextes, par ex. il l'aime à en être fou (Sandfeld 1965, 249). La délimitation des verbes causatifs peut être facilitée quelque peu par la description des «situations causatives» (cf. Shibatani 1976a, 1).

Le causatif morphologique se caractérise par la présence de morphèmes causatifs. Par ex. pour l'espagnol, Aranda Ortiz (1990, 159 sq) établit les suffixes -ificar (dulcificar), -izar (fertilizar), -ar (fecundar), -ecer (acontecer), etc. Le causatif morphologique est relativement fréquent dans les langues synthétiques. Ainsi, en hindi il montre une grande vitalité, comme en témoigne la constatation de Pobożniak (1979): «Das Causativum ist in der Hindi-Sprache eine reichlich entwickelte und produktive Kategorie, so daß man fast zu jedem Zeitwort ihre Form bilden kann. » Une telle vitalité n'est pas toujours le cas. Les causatifs morphologiques slaves, par exemple, sont différenciés, mais leur fréquence semble être beaucoup plus basse. Ainsi, Gołạb parle de trois types de causatifs slaves $(1968,93)$ qui se caractérisent par $1^{\circ}$ un suffixe et une apophonie, $2^{\circ}$ un suffixe, $3^{\circ}$ une déréflexivisation. Cependant, cette fréquence est relativement considérable, comme en fait foi l'existence de certains exemples considérés comme impossibles par des spécialistes. Ainsi Shibatani $(2002 \mathrm{~b}, 3)$ dit: "We are likely not to find a language in which causatives corresponding to verbs such as 'swim', 'sing' [...] are lexicalized». Pourtant de tels causatifs apparaissent dans les langues slaves, cf. pour le slovaque l'exemple de Heinisch (1977), possible aussi en polonais : Tento spevák vie rozespevat' celú salú 'Il sait faire chanter toute la salle'.

Les types morphologique et syntaxique servent à suppléer à la faible extension du lexique causal. De plus, le dernier type, permet «d'introduire une relation causale forte ou faible par la simple introduction d'un agent en position de sujet de l'opérateur faire ou laisser » (Moeschler 2003, 14). La construction en laisser, ou permissif, est souvent considérée comme un causatif à ceci près que sa force causative est faible (cf. p. ex. Simone -Cerbasi 2001, 446). 


\section{La tâche}

La majorité des travaux sur les constructions causatives concerne la syntaxe (ex. le passage d'un verbe intransitif au transitif), plus rarement la sémantique (ex. la causalité directe vs indirecte; cf. aussi Reboul 2003), rarement l'aspect communicatif (cf. Iliescu 1995, 3). On a étudié aussi l'aspect historique des constructions causatives romanes (cf. SimoneCerbasi 2001).

Dans notre article, nous nous limitons à la construction de base : faire + inf et incluons son synonyme fréquent et partiellement grammaticalisé dans les langues ibéro-romanes mandar (envoyer) + inf.

Nous nous assignons deux objectifs principaux. En premier lieu, nous essayons de déterminer la vitalité des constructions causatives en faire utilisées dans cinq langues romanes (l'espagnol, le français, l'italien, le portugais, le roumain), deux langues germaniques (l'allemand, l'anglais) et une langue slave (le polonais). Nous avons établi aussi la vitalité des équivalents, causatifs (lexicaux et morphologiques) et non causatifs, de ces constructions. Notre description se pose pour but $1^{\circ}$ de différencier les cinq langues romanes et $2^{\circ}$ de les opposer aux trois langues non romanes.

En deuxième lieu, nous essayons de déterminer à quel point la vitalité des constructions mentionnées s'explique par le caractère typologique des langues où elles sont utilisées.

Le premier de ces objectifs est moins important pour deux raisons: $1^{\circ}$ l'analyse des causatifs romans a fait l'objet de plusieurs études (p. ex. Iliescu 1995; Simone Cerbasi 2001) et $2^{\circ}$ l'explication est plus intéressante que la description.

\section{Le corpus}

Notre corpus embrasse cinq langues romanes : l'espagnol, le français, l'italien, le portugais et le roumain, deux langues germaniques: l'allemand et l'anglais, une langue slave: le polonais.

Le corpus I comporte presque 400000 mots graphiques pour chaque langue. Il se compose de 6 ouvrages dans leur intégrité (4 ouvrages français : CH, E, P, V, 1 ouvrage anglais: A et 1 ouvrage latin: IC), des trois premiers chapitres d'un ouvrage portugais (ML) et de fragments de deux ouvrages polonais (CD et $\mathrm{Q}$ ). Pour chaque ouvrage, nous disposons de l'original et de traductions en sept langues. Une exception: le texte CD n'a pas de version portugaise, ce qui nous a forcé à prendre en compte un 
fragment de la version portugaise d'un autre ouvrage (BA) de longueur équivalente à celle du texte manquant.

Trois de ces ouvrages sont rédigés dans une langue proche de la langue parlée: A, ML et CD. La langue de IC est quelque peu archaïsante.

Comme il n'est pas facile de manier cette masse d'informations, nous avons constitué un autre corpus, défini comme corpus II, utilisé surtout au tableau 1. Il se compose, pour chaque langue, de fragments des neuf ouvrages indiqués plus haut. De chaque ouvrage, nous avons retenu le fragment initial et le fragment final, chacun comportant, dans la version française, 2500 mots graphiques, à ceci près que nous ne coupons pas les phrases. Le corpus français comprend ainsi 45057 mots graphiques, au lieu de 45000 si les phrases avaient été coupées. Le corpus français une fois établi, nous avons constitué aussi le corpus des autres langues qui est à chaque fois composé des fragments équivalents. Ainsi, le corpus II comporte 45000 mots environ, quantité qui caractérise un roman moyen (cf. $\mathrm{CH}$ : presque 31000 mots, V: 76500 mots environ). Cependant, il ne permet pas de traiter certains problèmes, par ex. celui du causataire introduit par à ou par (cf. Marstrander 2000, 205).

\section{Occurrences des constructions causatives et de leurs équivalents}

Les verbes causatifs sont nombreux. Ainsi pour le polonais, Termińska $(1983,18)$ a accumulé un corpus de 500 verbes causatifs (= causatifs lexicaux) environ. Les occurrences des constructions causatives du type je fais le chien aboyer sont beaucoup plus rares. Pour rendre compte de la vitalité de ces constructions, nous nous fondons sur les occurrences qu'elles présentent dans des textes parallèles, tandis que la richesse des fonctions syntaxiques qu'elles assument, qui différencient peu les langues romanes, sera mentionnées plus bas $(\S \mathbf{6 . 4})$.

\subsection{Tableau des occurrences}

Le tableau 1 rend compte des constructions causatives du type faire + inf (roum. face + subjonctif) et de leurs équivalents: $1^{\circ}$ causatif lexical et causatif morphologique et $2^{\circ}$ équivalent non causatif. Nous retenons aussi l'équivalent ibéro-roman approximatif partiellement grammaticalisé du type mandar + inf; la valeur causative s'y confond en général avec la 
valeur lexicale de ce verbe (cf. Hernanz Carbó 2000, 2263). Par contre, nous n'avons pas retenu les verbes tels que prt. forçar, ang. bring, order, all. befehlen, roum. porunci, pol. kazać, fr. ordonner, etc., p. ex. il m'ordonne de partir au lieu de il me fait partir. Même en tant qu'équivalents du verbe faire dans sa fonction causative, ils sont tout au plus partiellement grammaticalisés, leur sémantique reste concrète.

Le verbe esp. prt. mandar est plus grammaticalisé que les verbes cités ci-dessus. On peut avancer un argument grammatical et un argument sémantique. Le premier c'est avant tout le comportement du pronom : il apparaît à l'accusatif avant mandar, tandis que devant des verbes tels que ordenar il apparaît au datif (pour plus de détails, cf. Hernanz Carbó op.cit., 2263-5). Un phénomène analogue se manifeste en principe aussi en portugais, p.ex.

(1) (a) fr. Le baron venait de le faire remettre à neuf $(\mathrm{V}, 35)$

(b) prt. barão acabava de o mandar arranjar de novo

(2) (a) prt. Só o mandarão entrar quando $(\mathrm{E}, 137)$

(b) prt. onde me mandou sentar (E, 95)

Le deuxième, c'est la sémantique généralisée de mandar qui apparaît par ex. dans

(3) (a) fr. On voulut sonner le domestique pour faire avancer la voiture. (V, 117)

(b) esp. Quisieran llamar el criado para que hiciese adelantar el carruaje.

(c) prt. Quiseram tocar, a chamar o criado, para que este mandasse avançar a carruagem.

(d) it. Si volle richiamare il servitore per far avvicinare la carrozza.

(4) (a) prt. Alguns passos adiante, mandam-me parar e perguntam-me se aquela mulher é minha Mãe. (ML, 81)

(b) fr. Quelques pas plus loin, ils me firent arrêter.

(c) esp. Algunos pasos más adelante, me mandaron parar y me preguntaron.

Il y a un certain nombre de différences entre la construction mandar + inf en espagnol et en portugais.

Une différence qui saute aux yeux c'est la fréquence d'emploi. La construction du type mandar + inf apparaît 1 fois dans les versions espagnoles de $\mathrm{A}$, de $\mathrm{P}$, de $\mathrm{IC}$ et de $\mathrm{V}, 0$ dans $\mathrm{CH}$ et dans $\mathrm{E}$. Il en va tout autrement des versions portugaises: 19 occurrences dans V, 17 dans $\mathrm{P}$, 
10 dans A, 9 dans E, 2 dans CH, 1 dans IC. Dans ML il s'agit probablement de l'influence de l'original sur la traduction: 47 occurrences dans l'original portugais et 46 en espagnol.

Le subjonctif après mandar peut apparaître dans les deux langues, il est plus fréquent en espagnol, par ex.

(5) (a) prt. Mandei-a, então, dar-lhe um abraço e um beijo. (ML, 22)

(b) esp. Le mandé que le diera un abrazo y un beso.

(6) (a) fr. Malgré le froid, la baronne fit apporter un siège afin de le regarder travailler. (V, 113)

(b) prt. Apesar do frio, a baronesa mandou que lhe trouxessem uma cadeira.

(c) it. Nonostante il freddo, la baronessa fece portare una sedia.

Ainsi, il y a un certain rapport entre la quantité des constructions causatives et celle des subjonctifs (cf. aussi Iliescu 1995, 11).

Pour le roumain, on signale, à côté du tour face + subjonctif, aussi la construction pune + subjonctif (cf. idem.). Ces tours sont en grande partie grammaticalisés. Cependant le degré de grammaticalisation est moins élevé en roumain que dans les autres langues romanes. Pour certaines langues on se demande s'il n'est pas légitime de parler d'un seul verbe causatif, par ex. Comrie constate $(1976,296)$ : «the analytic causative in certain languages, such as the French causative with faire [...] functions in many respects like a fused verbal construction.» Or, une telle constatation n'est pas motivée pour le roumain où le verbe face est suivi d'une conjonction qui lui assure une certaine autonomie. Un objet sépare facilement le verbe face et le subjonctif, comme dans

(7) (a) fr. Arrivé là, on admettra sans peine que rien ne pouvait faire espérer à nos concitoyens les incidents qui se produisirent au printemps de cette année-là. $(\mathrm{P}, 7)$

(b) esp. Siendo así las cosas, se admitirá fácilmente que no hubiese nada que hiciera esperar a nuestros conciudadanos los acontecimientos que se produjeron a principios de aquel año.

(c) it. Qui giunti, si ammetterà senza fatica che nulla poteva far presagire ai nostri concittadini gli incidenti che si verificarono nella primavera di quell'anno.

(d) prt. Uma vez lá, admitir-se-á sem custo que nada podia fazer esperar aos nossos concidadãos os incidentes que se produziram na Primavera desse ano.

(e) roum. Ajunşi aici, vă fi lesne să admitem că nimic nu i putea face pe concetătenii noştri să prevadă incidentele care au avut loc în primăvara anului acela.

Acta Linguistica Hungarica 53, 2006 
Le tour roum. pune + inf est présenté par Iliescu (1993). Il ne ressort pas de ce travail que la construction du type face + subjonctif jouit d'une richesse comparable à celle des constructions ibéro-romanes en hacer/ fazer, tandis que le tour roum. en pune apparaît sporadiquement. Ainsi dans notre corpus II, il apparaît 3 fois à peine (la construction en face 25 fois). Dans V on a 68 constructions en face et 6 en pune. Dans E, on trouve 6 constructions en pune, dans $\mathrm{A}-5$, dans $\mathrm{CH}-2$.

Les langues germaniques offrent quelques constructions causatives. Duffley $(1992,57)$ parle des constructions anglaises en make, cause et have. Or, leurs occurrences dans notre corpus sont peu nombreuses: la construction en cause apparaît 12 fois dans tout notre corpus I ( 7 dans IC, p. ex. the love of Jesus causes man to regard himself very humbly), celle en have n'apparaît jamais comme équivalent de fr. faire causatif dans V. Il en va de même de all. heißen qui forme trois constructions causatives dans notre corpus I (ex. führte man mich mit dieser Dame in einen Saal und hiess uns ein wenig warten-ML).

Tableau 1

Occurrences des constructions causatives introduites par fr. faire, envoyer; esp. hacer, mandar; it. fare; prt. fazer, mandar ; roum. face; ang. make; all. lassen et de leurs équivalents dans le corpus II

\begin{tabular}{lcccccccc}
\hline \multicolumn{1}{c}{ Langue } & FR & ESP & IT & PRT & ROUM & ANG & ALL & POL \\
Forme & & & & & & & & \\
\hline $\begin{array}{l}\text { Construction } \\
\text { faire + inf }\end{array}$ & 48 & 28 & 39 & 26 & $* 28$ & 12 & 30 & 0 \\
$\begin{array}{l}\text { Causatif } \\
\text { synthétique }\end{array}$ & 13 & 18 & 13 & 13 & 9 & 24 & 8 & 27 \\
$\begin{array}{l}\text { Construction } \\
\text { envoyer +inf }\end{array}$ & 2 & 5 & 1 & 8 & 2 & 3 & 2 & 9 \\
\hline $\begin{array}{l}\text { Total formes } \\
\text { causatives }\end{array}$ & 63 & 51 & 53 & 47 & 39 & 39 & 40 & 36 \\
$\begin{array}{l}\text { Équivalent } \\
\text { non causatif } \\
\text { Total }\end{array}$ & 12 & 24 & 22 & 28 & 36 & 36 & 35 & 39 \\
\hline
\end{tabular}

*_le tour roum. face/pune + subjonctif; causatif synthétique $=$ causatif lexical et, très rarement, causatif morphologique

Comme au tableau 1 nous n'avons pas retenu tous les verbes causatifs, mais seulement ceux qui ont pour équivalent au moins dans une autre langue une construction causative, le tableau ne permet pas de déterminer le rôle qui revient à trois types de causatif: lexical, morphologique et 
syntaxique dans l'expression de la causalité. Cependant, il jette quelque lumière sur ce problème vu qu'on a tenu compte aussi des deux types d'équivalents: causatif et non causatif. Nos conclusions les plus importantes sont les suivantes. La tendance générale à envisager une action comme causative est la plus forte en français. Elle est un peu plus faible dans les autres langues romanes, le roumain excepté. Elle est la plus faible en roumain et dans les langues non romanes.

Les données relatives à la construction allemande en lassen sont peu süres, le même auxiliaire servant aussi à former le permissif.

L'emploi de la construction causative est facilitée par la mise en relief de la valeur causative, comme dans

(8) (a) fr. S'il s'en est servi, c'est seulement pour comprendre ou faire comprendre ses concitoyens et pour donner une forme, aussi précise que possible, à ce que, la plupart du temps, ils ressentaient confusément. $(\mathrm{P}, 249)$

(b) esp. Si se sirvió de ella fue solamente por comprender o hacer comprender a sus conciudadanos.

(c) it. S'egli se n'è servito, è soltanto per capire o far capire i suoi concittadini.

(d) prt. Se se serviu delas, foi apenas para compreender ou fazer compreender os seus concidadãos.

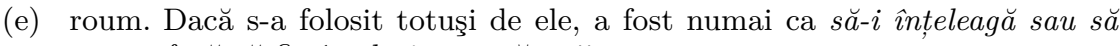
facă să fie înțeleşi concetățenii.

(f) pol. Tylko po to, żeby rozumieć lub wytłumaczyć swych współobywateli.

Seul le polonais oppose les deux sens, causatif et non causatif, sans dépasser le niveau lexical.

En ce qui concerne la construction du type faire + inf, seul le français se sépare nettement des autres langues, relativement peu de l'italien. Les langues qui restent se divisent en trois catégories: $1^{\circ}$ l'espagnol, le portugais, le roumain et l'allemand, $2^{\circ}$ l'anglais et $3^{\circ}$ le polonais. Les langues appartenant à la première catégorie sont plus pauvres que les langues «du centre»: le français et l'italien. La prise en compte des rares exemples de esp. prt. mandar + inf ne change pas la conclusion générale. La faiblesse relative de la construction causative anglaise devrait étonner vu le caractère analytique de cette langue. La construction du type «accusativus cum infinitivo», dont la construction causative est un cas particulier, n'a pas survécu en polonais et les tentatives de la réintroduire ont échoué (cf. Urbańczyk-Kucała 1999, 11). 


\subsection{Deux autres enquêtes}

En vue de parer aux faiblesses du corpus II, nous procédons encore à deux enquêtes destinées à confirmer la vitalité des constructions en faire. La première (tab. 2) est fondée sur deux ouvrages entiers: A et E. Ici, nous n'indiquons pas la construction roumaine du type face + subjonctif.

\section{Tableau 2}

Occurrences des constructions causatives romanes en faire (aussi envoyer, esp. prt. mandar) + inf sur la base de deux ouvrages

\begin{tabular}{lcccccc}
\hline Forme & $\begin{array}{c}\text { Langue } \\
\text { Euvre }\end{array}$ & FR & ESP & IT & PRT & ROUM \\
\hline Construc. & A & 51 & 16 & 35 & 20 & 0 \\
causative & E & 32 & 30 & 38 & 12 & 0 \\
Total & & 83 & 46 & 73 & 32 & 0 \\
\hline
\end{tabular}

Les données réunies au tableau confirment celles du tableau 1. Les constructions causatives françaises et italiennes sont plus riches que les constructions causatives espagnoles, portugaises ou roumaines. Les formes roumaines face + inf ne sont pas représentées dans les deux tableaux, mais Iliescu $(1995,11)$ constate que l'infinitif, au lieu du subjonctif, peut apparaître exceptionnellement.

La deuxième enquête porte sur le roman Une vie de Maupassant : 116 constructions causatives françaises en faire, 76 italiennes, 68 constructions roumaines du type face + subjonctif et 2 du type pune + subjonctif, 119 constructions allemandes en lassen, dont 70 environ sont des causatifs propres (les autres, des permissifs). Elle a confirmé aussi la faiblesse de la construction anglaise make + inf: dans les premières 1000 phrases de V, on trouve 33 constructions françaises en faire et seulement 12 anglaises en make.

L'enquête est motivée entre autres par l'opinion avancée par Iliescu $(1995,12)$ : «Pour le roumain, l'emploi de a face comme auxiliaire factitif est souvent exclu». Or, notre enquête, qui ne concerne pas les formes acceptables mais seulement utilisées dans un texte, apporte une certaine contribution à ce problème: numériquement, les constructions roumaines ne s'avèrent pas plus pauvres que les constructions ibéro-romanes et dans $\mathrm{V}$, elles sont aussi riches que les constructions italiennes. Iliescu aurait dû préciser les catégories de constructions françaises ou italiennes dont les équivalents roumains fidèles sont impossibles. Notre corpus permet de 
signaler l'absence du tour roumain équivalent au tour fr. se faire + inf, qui ne semble pas être causatif, par ex.

(9) (a) fr. Le froid du matin commençait à se faire sentir dans la pièce. (P, 235)

(b) esp. empezaba a hacerse sentir en la habitación.

(c) it. Il freddo della mattina cominciava a farsi sentire nella stanza.

(d) prt. O frio da manhã começava a fazer sentir-se no quarto.

(e) roum. Frigul dimineții începea să se facă simțit în odaie.

Un autre problème c'est la vitalité de la construction causative italienne du type fare + inf. Différents chercheurs constatent une similitude poussée entre le français et l'italien. Ainsi Iliescu $(1995,12)$ écrit: «le français et l'italien se comportent d'une façon étonnante presque de la même façon, ce qui est confirmé par la totalité des exemples trouvés dans la traduction du livre de Simenon».

À leur tour, Simone et Cerbasi $(2001,441)$ disent: «Italian and French are strongly causative-oriented languages, i.e., they tend to see facts as causatively structured to an extent that is probably unique among the Romance languages.»

En dépit de ces opinions, nos tableaux montrent qu'il y a une différence numérique entre les constructions causatives dans les deux langues. Aussi bien notre tableau 1, basé sur 9 ouvrages dont 4 seulement sont français (48 ex. fr. contre 39 ex. it.), que les données offertes par un ouvrage anglais (A : 51 ex. fr. contre 35 ex. it.) et par un ouvrage français (V : 116 ex. fr. contre 76 ex. it.) mettent en évidence une différence nette entre les deux langues. Les données différentes offertes par un seul ouvrage ( $\mathrm{E}: 38$ constructions causatives italiennes contre 32 françaises) ne changent pas la conclusion générale. Cette conclusion est corroborée par des facteurs autres que numériques (cf. ci-dessous, §5).

\section{Causalité directe et indirecte}

Une définition très simple de Shibatani et Pardeshi $(2002,88)$ nous suffit: «Therefore it is a good first approximation to define direct causative and as a situation involving an agentive causer and a patientive causee and indirect causation as one involving two agentive participants, one an agentive causer and the other an agentive causee.» 
En principe, le verbe simple désigne une causation directe, la construction en faire - une causation indirecte. Cependant, la causation lexicale est ambiguë vu que le verbe simple peut dénoter assez souvent aussi bien une causation directe qu'indirecte: J'ai réparé ma voiture peut signifier soit 'je l'ai réparée moi-même' soit 'j'ai fait réparer ma voiture par un mécanicien'. La construction causative en faire est ambiguë elle aussi $\mathrm{vu}$ qu'elle peut dénoter aussi bien une causation indirecte que directe (ex. il fit remarquer $-1^{\circ}$ il dit, $2^{\circ}$ il fit qu'un autre remarqua). Une autre ambiguïté est signalée par Robustelli $(1995,203)$. Une phrase telle que Faccio portare il fratello peut avoir deux interprétations: $1^{\circ}$ Faccio che $i l$ fratello porti et $2^{\circ}$ Faccio che il fratello sia portato.

Dans un certain nombre d'exemples, seul le français exprime une causalité indirecte, c'est-à-dire qu'il emploie plus de constructions du type faire + inf que les autres langues prises en compte, y compris l'italien. Dans l'exemple qui suit, aussi bien l'original polonais que les traductions emploient un verbe simple même si la causalité relative à l'action d'enlever est indirecte. Seul le traducteur français introduit la construction en faire, plus appropriée dans ce cas:

(10) (a) pol. Zamiast pokłonić się o nią Aulusowi i Pomponii, odebrat dziecko podstępem rodzicom. (Q, 88)

(b) fr. Au lieu de se présenter devant Aulus et Pomponia pour la leur demander, il l'avait fait enlever par subterfuge.

(c) esp. les había arrebatado la hija valiéndose de astucias

(d) it. aveva strappato con un inganno la figlia ai genitori

(e) prt. arrebatara-a, de surpresa, aos seus pais adoptivos

(f) roum. o răpise prin surprindere, părinților ei

(g) all. hatte er sie entführt durch List

Dans cet exemple, la connaissance du contexte précédent permet de constater que Vinicius n'a pas enlevé lui-même la femme en question, par conséquent l'emploi de la construction causative en français est plus régulier que l'emploi du verbe simple dans les autres langues qui procèdent à l'«effacement en surface».

Dans l'exemple suivant, où il s'agit d'une causalité directe, l'original portugais présente un causatif lexical, qui est retenu dans les traductions sauf en français:

(11) (a) prt. E quem melhor que este Imaculado Coração nos poderia descobrir os segredos da Divina Misericórdia? (ML, 16) 
(b) fr. Et qui d'autre que le Coeur Immaculé pourrait faire découvrir les secrets de la Miséricorde Divine?

(c) esp. Y, ¿quién mejor que este Inmaculado Corazón nos podría descubrir los secretos de la divina Misericordia?

(d) it. E chi meglio di questo Cuore Immacolato ci potrebbe scoprire i segreti della Misericordia Divina?

(e) roum. Şi cine alta decât Neprihănita Inimă ar putea să dezvăluie tainele îndurării divine?

(f) ang. could have revealed to us the secrets of Divine Mercy?

(g) pol. odkryć tajemnice Boskiego Miłosierdzia?

(h) all. die Geheimnisse der göttlichen Barmherzigkeit aufdecken?

Dans presque toutes ces versions, il s'agit d'une seule action (de découvrir certains secrets) accomplie par un seul agent et non pas d'inciter un autre agent à le faire. Seul le français emploie une construction du type faire + inf et envisage l'action comme une incitation à découvrir certains secrets. Dans les autres langues cela se produit plus rarement. Ici il convient de signaler des tours tels que faire remarquer, faire observer qui, en dépit de leur caractère analytique, se rapportent souvent à une causalité directe.

Ces formes analytiques sont possibles non seulement en français, mais dans cette langue elles sont les plus fréquentes, par ex.

(12) (a) fr. Il aurait pu recommencer, comme le lui fit remarquer Rieux. (P, 71)

(b) esp. como le decía Rieux

(c) it. come gli osservò Rieux

(d) prt. com lhe fez notar Rieux

(e) roum. cum l-a făcut să observe Rieux

(f) ang. as Rieux pointed out

(g) all. wie Rieux es ihm riet

(h) pol. ak zauważyt Rieux

Les langues non romanes emploient des équivalents synthétiques; voir encore

(13) (a) fr. Mais d'autres sont morts, fit remarquer Rieux. (P, 44)

(b) esp. hizo observar Rieux

(c) it. fece notare Rieux

(d) prt. fez notar Rieux

(e) roum. atrage atenția Rieux 
(f) ang. Rieux observed

(g) all. bemerkte Rieux

(h) pol. zauważyt Rieux

L'italien est un peu plus pauvre que le français. Une différence se manifeste quand l'original n'est pas roman. Dans A on trouve 15 exemples français (10 du type faire observer et 5 du type faire remarquer) et 0 italiens, dans $\mathrm{Q}-2$ exemples français et 0 italiens, mais dans CD 0 pour les deux langues.

Ainsi, la réalisation de l'opposition causalité directe/causalité indirecte apporte un argument de plus en faveur de la thèse qu'on ne devrait pas traiter sur un pied d'égalité le français et l'italien (cf. aussi ci-dessous, $\S$ 6.1) comme on le fait parfois (cf. Iliescu 1995, 12). Notre corpus conduit à différencier les deux langues.

\section{Remarques typologiques}

Les langues romanes sont liées non seulement par l'origine commune, mais aussi par un certain complexe de traits typologiques. Rien d'étonnant à ce que certains parlent d'un type roman (par ex. Coşeriu 1988). Nous avons montré à plusieurs reprises que les langues romanes se distinguent des langues non romanes prises en compte ici par le degré de synthétisme/ analytisme de cette façon que le polonais et, dans une mesure plus limitée, l'allemand sont plus synthétiques, l'anglais - plus analytique que les langues romanes (cf. Gawełko 2001, 40). Il n'est pas sans intérêt de déterminer si la construction analytique en faire (all. lassen, ang. make) est effectivement plus riche en anglais et moins riche en allemand et en polonais que dans les langues romanes.

On peut généraliser cette tâche et proposer de déterminer le rapport entre la vitalité de la construction en faire et la tendance analytique. Une telle tâche est légitime vu que des spécialistes de typologie universelle présentent les formes causatives dans le cadre d'une échelle qui va de la forme synthétique vers la forme analytique, comme dans GivónYoung $(2002,52)$ : lexical causative (She broke the window), morphological causative (She enlarged the house), co-lexicalization (She let-go of his hand), non-finite complementation (She made him leave), subjunctive complementation (She asked that he leave). Ils constatent à propos de cette série de formes: «The causative constructions at the top of 
the complementation scale display the highest level of clause integration, code the strongest and the most direct causation, and are the most likely to involve a non-human patient as the causee. Toward the bottom of the scale, constructions display lower levels of clause union, code weaker and/or less-direct causation, are more likely to have a human agent as the causee» (op.cit., 51).

Notre corpus II ne peut que confirmer ce schéma, notamment en ce qui concerne l'agent $1^{\circ}$ d'un causatif lexical et $2^{\circ}$ d'un infinitif introduit par faire. Or, le causatif lexical comporte un agent animé ou inanimé dans des proportions rapprochées, tandis que dans la construction en faire l'agent animé de l'infinitif prédomine largement : 30 agents animés contre 18 inanimés dans le texte français, 8 animés contre 4 inanimés dans le texte anglais.

Dans les langues du monde une distinction semble se dessiner: le causatif analytique apparaît de préférence dans les langues analytiques tandis que les langues synthétiques ont recours à des affixes (cf. Shibatani 1976b, 2-3). Ce principe trouve une confirmation en latin où, à en croire D. Strong (cf. Simone-Cerbasi 2001, 450), on observe un passage de causatifs morphologiques aux causatifs syntaxiques.

La question se pose de savoir si, dans les langues prises en compte, la distribution des formes causatives les plus importantes est conforme au caractère typologique de ces langues.

\subsection{Le français}

La forte tendance analytique du français semble expliquer la grande vitalité des constructions causatives en faire, observée aux $\$ \S 4$ et $\mathbf{5}$. Cette tendance explique donc les occurrences de la construction française faire + inf et les cas particuliers : $1^{\circ}$ l'emploi régulier de cette construction dans le cas d'une causalité indirecte, tandis que les autres langues tendent à employer dans ce cas un causatif synthétique (lexical) et $2^{\circ}$ l'emploi de la construction faire + inf dans le cas d'une causalité directe, phénomène plus rare dans les autres langues.

\subsection{Les autres langues romanes}

À lire ce qui précède, un doute surgit : l'italien comporte une construction causative analytique qui se rapproche de celle en français et pourtant 
sa tendance analytique est beaucoup plus faible que celle du français. Or, la marche vers l'analytisme, qui caractérise bien les langues indoeuropéennes, n'est pas un processus homogène. L'infinitif italien évolue relativement vite. Telle sa tendance nominale: au cours de son histoire l'infinitif italien gagne en caractère nominal. Les informations apportées par Vanvolsem (1983, 168 tab.) confirment cette remarque : l'infinitif est sensiblement plus nominal chez Manzoni (XIX ${ }^{\mathrm{e}}$ s.) que chez Boccaccio $\left(\mathrm{XIV}^{\mathrm{e}} \mathrm{s}\right.$.).

Cependant, cet argument a une valeur très limitée. La force de la tendance analytique est très rapprochée dans les langues espagnole, italienne et portugaise, tandis que la vitalité de la construction en faire différencie ces langues d'une façon notable: l'italien se sépare nettement des langues ibéro-romanes en se rapprochant du français.

L'explication de la forme roumaine est relativement simple. La construction causative la plus fréquente face + subjonctif est en rapport avec la faiblesse de son infinitif, laquelle est un trait balkanique. Ainsi, c'est avant tout la géographie linguistique qui explique la tendance à remplacer l'infinitif par le subjonctif.

\subsection{L'allemand}

L'explication de la fréquence relativement considérable de la construction allemande est plus difficile. Une explication partielle est que la tendance analytique de l'allemand est plus forte qu'elle ne paraît de prime abord. La déclinaison des substantifs, qui donne l'impression d'une langue hautement synthétique, y est faible. L'opposition entre le nominatif et l'accusatif ne persiste qu'au masculin singulier. On trouve des formes caractéristiques des langues analytiques, par ex. l'article, le pronom sujet obligatoire, le présentatif du type es gibt. On note de plus la faiblesse de l'aspect verbal, la force du passif, etc. (cf. Gawełko 2001, 29). Ainsi, la fréquence considérable du causatif analytique en lassen ne devrait pas nous étonner excessivement.

\subsection{L'anglais}

L'irrégularité la plus importante c'est la faiblesse relative de la construction anglaise du type make + inf. Avant d'en proposer une explication, il faut prendre position par rapport à certaines opinions. D'après cer- 
tains chercheurs, l'infinitif anglais en général aussi bien que sa proposition infinitive sont forts. Ainsi Rémi-Giraud est d'avis que, à l'opposé du français et de l'allemand, «l'anglais et le grec connaissent une grande extension de la proposition infinitive» $(1988,59)$. L'argument est apporté par la richesse du «système d'opposition aspectuelle». L'anglais présente un «système à quatre formes» (to make, to have made, to be making, to have been making). Par contre, ne disposant que de deux formes (fr. dormir et avoir dormi, all. schlafen et geschlafen haben), l'infinitif est considéré comme faible en français et en allemand.

Ainsi, pour déterminer la vitalité d'une construction, Rémi-Giraud se fonde sur la complexité d'oppositions formelles. D'autres chercheurs, au contraire, considèrent les occurrences d'une forme comme facteur déterminant sa vitalité. Ainsi Simone et Cerbasi $(2001,447)$ constatent que «Latin [...] is able to see facts causatively, and, accordingly, does feature several types of CCs [causative constructions], but nonetheless exhibits a surprisingly low frequency of CCs.» Et plus tard (p. 450) : «it was a relatively poor causative-oriented language.» Alors, en dépit de l'existence d'un certain nombre de constructions causatives, les auteurs considèrent le latin comme «a poor causative-oriented language».

Nous pensons qu'en effet c'est la fréquence de différentes constructions dans les textes, telles les constructions causatives du type faire/ mandar + inf, qui est le critère de base déterminant leur vitalité. À l'occasion on peut rappeler que les vieux textes français offraient une richesse des mots. Cependant une telle richesse est illusoire tant qu'elle n'est pas fondée sur la fréquence des mots et sur la richesse des sens qu'ils expriment. Nous partons donc de la conception que la richesse des constructions se manifeste, en premier lieu, par leur fréquence dans les textes ainsi que, en deuxième lieu, par la richesse des fonctions syntaxiques qu'ils assument.

En ce qui concerne la fréquence, il convient d'ajouter les données relevées dans $\mathrm{A}$ et $\mathrm{E}$ que l'on peut comparer avec celles réunies au tableau 2: 15 constructions du type make + inf dans A et 16 dans E. Ainsi, les constructions françaises sont deux fois plus nombreuses dans $\mathrm{E}$ et trois fois dans A. On s'aperçoit que l'original anglais de A ne favorise pas la quantité des exemples anglais.

On peut conclure que, compte tenu des deux facteurs: fréquence dans les textes et diversité des fonctions syntaxiques, la construction anglaise make + inf s'avère plus faible que ne le laisse suggérer la tendance analytique de cette langue. 
En somme, l'infinitif anglais, y compris la construction du type make + inf, est relativement faible. Cette faiblesse est à rechercher dans la concurrence des formes en -ing, non finies elles aussi. La faiblesse de la construction causative en make est un facteur qui plaide en faveur de la faiblesse de l'expression causative de l'anglais. Cependant étant donné la force de ses causatifs lexicaux, on ne peut pas dire qu'il soit «a poor causative-oriented language».

\subsection{Le polonais}

La faiblesse de la tendance analytique explique l'absence de la construction causative analytique en polonais. Le fait que différentes langues slaves, y compris le polonais, ont employé d'une façon limitée cette construction n'a rien d'irrégulier non plus.

\subsection{Coefficient de similitude}

Nous avons signalé à plusieurs reprises la plus grande richesse des constructions causatives françaises en faire mais aussi une position privilégiée de l'italien. Le coefficient de similitude rend compte de ce fait. Il est la quantité des exemples réunis au tableau 1 dans lesquels deux langues comparées (le français et l'espagnol, le française et l'italien, etc.) comportent un causatif en faire. Elle est de 29 pour le français et l'italien. Cette quantité paraît basse: (29 sur un total de 75 ; cf. les remarques de Iliescu $(1995,12)$ sur les traductions italiennes des causatifs français employés dans un livre de Simenon). Cela tient à la façon de compter les exemples. Nous retenons les exemples même dans le cas où une seule langue, pas nécessairement celle de l'original, comporte une construction en faire, tandis que les autres langues offrent des équivalents tels que causatif lexical, construction avec un verbe non causatif ou causatif mais non grammaticalisé (ex. ordonner au lieu de faire).

Ce coefficient est presque identique pour le français comparé à l'espagnol et au portugais : respectivement de 18 et de 17 exemples. Si l'on prenait en compte la construction causative en mandar, le coefficient pour le français et le portugais s'élèverait à 20. La similitude entre le français et le roumain est relativement grande (15) à ceci près que le roumain emploie le subjonctif au lieu de l'infinitif. La similitude entre le français et l'allemand s'élève à 10 exemples comportant la construction analytique, 
celle entre le français et l'anglais à 7 . Ces données permettent de constater que $1^{\circ}$ la similitude entre le français et l'italien est la plus grande et que $2^{\circ}$ la similitude entre le français et les langues romanes est plus grande que celle entre le français et les langues non romanes. Ici c'est le critère génétique qui prédomine: les langues romanes du centre, le français et l'italien, s'avèrent les plus rapprochées, tandis que les langues non romanes - les plus éloignées du français. La faiblesse du critère de similitude est qu'il montre un rapport étroit avec la fidélité des traductions, la plus facile à atteindre entre le français et une autre langue romane.

\section{Remarques finales}

L'article comporte deux éléments essentiels: $1^{\circ}$ établissement de la vitalité des constructions causatives dans les cinq langues romanes les plus connues, deux langues germaniques et une langue slave sur la base d'un corpus de textes parallèles et $2^{\circ}$ explication typologique des différences observées.

La vitalité des constructions causatives est la plus grande en français, un peu moins grande en italien. La catégorie suivante est formée par les constructions des autres langues romanes et de l'allemand. Les constructions anglaises sont plus pauvres. Le polonais de nos jours n'a pas de construction causative analytique.

Avant de proposer une explication typologique des différences observées, il convient de rappeler les catégories qui se dégagent de notre classification des langues prises en compte (Gawełko 2001, 40) : $1^{\circ}$ l'anglais et le français, $2^{\circ}$ les autres langues romanes, $3^{\circ}$ l'allemand et $4^{\circ}$ le polonais. Ces catégories vont des langues les plus analytiques vers les plus synthétiques. Les langues appartenant à la deuxième catégorie se différencient aussi par le degré plus ou moins élevé d'analytisme, mais les différences qu'elles offrent ne vont pas loin et l'influence qu'elles exercent sur la spécificité des catégories grammaticales est limitée.

Le principe général est que la construction causative analytique augmente son importance avec l'augmentation du caractère analytique de la langue où elle est employée. Les quatre catégories rappelées ci-dessus expliquent le mieux le comportement $1^{\circ}$ du français: la grande richesse des constructions causatives en faire est en rapport avec le haut degré de son caractère analytique et $2^{\circ}$ du polonais : le haut degré de synthétisme est en rapport avec l'absence de construction causative. Le comportement des langues ibéro-romanes et du roumain est régulier dans ce sens 
que leurs constructions causatives sont plus faibles que celles en français, mais plus riches que celles en polonais.

L'irrégularité la plus importante est offerte par l'anglais: le haut degré d'analytisme n'est pas en accord avec la faiblesse relative de ses constructions causatives. La faiblesse en question est en rapport avec la faiblesse de son infinitif, concurrencé par les formes en -ing.

Le comportement de l'allemand n'est pas tout à fait régulier : il est plus synthétique que n'importe quelle langue romane et pourtant la vitalité de ses constructions causatives se rapproche de celle des constructions de la majorité des langues romanes. Nous avons proposé une explication partielle de ce fait en constatant que la différence dans le degré de synthétisme/analytisme entre la majorité des langues romanes et l'allemand ne va pas très loin.

L'irrégularité présentée par l'italien consiste en ce que son caractère typologique se rapproche de celui des langues ibéro-romanes (cf. Gawełko 2001, 40) et pourtant ses constructions causatives - des constructions françaises. Pour jeter quelque lumière sur ce fait, il convient de constater que les langues appartenant à une famille présentent parfois un comportement similaire en dépit de différences dans le degré de synthétisme/ analytisme qui les caractérise. Telle la vitalité de l'infinitif, similaire dans les langues romanes, le roumain excepté. Or, en principe cette vitalité tient aussi au degré d'analytisme de la langue où il est employé. Cependant c'est l'infinitif portugais qui est un peu plus fort que l'infinitif des autres langues romanes.

Les langues ibéro-romanes et le roumain ne montrent pas d'irrégularité. Cependant chaque langue présente une certaine spécificité.

Le roumain offre un cas particulier : l'élimination progressive de l'infinitif, laquelle est un trait balkanique, entraîne la construction causative du type face + subjonctif.

La faiblesse relative de la construction portugaise fazer + inf peut étonner vu que l'infinitif portugais est le plus fort dans la Romania. Cette faiblesse relative est en rapport avec le caractère très verbal de l'infinitif portugais : sujet syntaxique relativement fréquent, p. ex. o tempo de ela partir para Lisboa (ML, 86); présence du sujet flexionnel, très rare dans les langues du monde, p. ex. o amigo ficaria contente por chegarmos tão cedo (E, 82) ; substantivation relativement rare (cf. Disterheft 1980, 198; Gawełko 2004). 


\section{Corpus}

$1^{\circ}$ Camus, A. L'étranger, Paris, Gallimard, 1942 ; El extranjero, Madrid, El Libro de Bolsillo, 1982; Lo straniero, Milan, Tascabili Bompiani, 1988; O estrangeiro, Lisbonne, Unibolso, s.d.; Străinul, in Străinul, Ciuma, Căderea, Exilul şi împărătia, Bucarest, RAO International Publishing Company, 27-92, 1993; The Outsider, London, Penguin Books, 1983; Der Fremde, Reinbeck bei Hamburg, Rowohlt, 1993; Obcy, Varsovie, Krąg, 1991. (E)

$2^{\circ}$ Camus, A. La chute, Paris, Gallimard, 1989; La caída, Madrid, Alianza Editorial, 1982; La caduta, Milan, Tascabili Bompiani, 1989; A Queda, Lisbonne, Livros do Brasil e Editorial Verbo, 1971; Căderea, in Străinul, Ciuma, Căderea, Exilul şi împărătia, Bucarest, RAO International Publishing Company, 295-369, 1993; The Fall, London, Penguin Books, 1963; Der Fall, Reinbeck bei Hamburg, Rowohlt, 1995 ; Upadek, Varsovie, Krąg, 1991. (CH)

$3^{\circ}$ Camus, A. La peste, Paris, Gallimard, 1989; A peste, Lisbonne, Livros do Brasil, s.d. ; La peste, Barcelone, Edhasa, 1977 ; La peste, Milan, Tascabili Bompiani, 1989 ; Ciuma, in Străinul, Ciuma, Căderea, Exilul şi ı̂mpărăția, Bucarest, RAO International Publishing Company, 93-294, 1993; The plague, London, Penguin Books, 1960 ; Die Pest, Hambourg, Rowohlt, 1994; Dżuma, Varsovie, Jota, 1991. (P)

$4^{\circ}$ Maupassant, G. de. Une vie, Paris, Gallimard, 1974; Una vida, in G. de Maupassant, Obras completas, 149-285, Madrid, Aguilar, 1965; Uma vida, Mem Martins, Livros de bolso Europa-America, 1974; Una vita, Farigliano, Arnoldo Mondadori Editore, 1984; O viață, Chişinău, Făt-Frumos, 1994; A Woman's Life, London, Penguin Books, 1965 ; Ein Leben, Frankfurt/Main \& Berlin, Ullstein, 1995; Historia jednego życia, Varsovie, Zrzeszenie Księgarstwa, 1985. (V)

$5^{\circ}$ Memórias da Irmã Lúcia, Fátima, Postulação, 1978; Mémoires de Sœur Lucie, Fátima, Postulação, 1980; Memorias de la Hermana Lucía, Fátima, Postulação, 1978; Memorie di Suor Lucia, Fátima, Postulação, 1980 ; Sora Lucia despre Fatima, Fátima, Secretariado dos Pastorinhos, 2002 ; Fatima in Lucia's own words, Fátima, Postulação, 1976 ; Schwester Lucia spricht über Fátima, Fátima, 1975 ; Siostra Lucja mówi o Fatimie, Fátima, Postulação, 1978 (jusqu'à la p. 104 de l'original portugais). (ML)

$6^{\circ}$ Tomas a Kempis, De imitatione Christi; (O naśladowaniu Jezusa Chrystusa), édition bilingue, Varsovie, Instytut Wydawniczy Pax, 1981; L'imitation de JésusChrist, Paris, Édition du Seuil, 1961; L'imitazione di Cristo, Milan, Edizioni Paoline, 1988; Imitación de Cristo, Barcelone, Editorial Regina, 1987 ; Imitação de Cristo, Braga, Editorial Franciscana, 1980 ; Urmarea lui Hristos, Timişoara, Editura Metropoliei Banatului, 1991; The Imitation of Christ, London, Penguin Books, 1965; Thomas von Kempen, Die Nachfolge Christi, Kevelaer, Butzon \& Bercker, 1990; Tomasz à Kempis, Naśladowanie Chrystusa, Cracovie, WAM, 2000. (IC)

$7^{\circ}$ Carrol, L. Alice's adventures in Wonderland-Les aventures d'Alice au Pays des merveilles, Paris, Aubier-Flammarion, 1970; Aventuras de Alicia en el país de las maravillas, Barcelone, Moby Dick, 1973; Alice nel Paese delle Meraviglie, in Alice nel Paese delle Meraviglie, Attraverso lo specchio, Milan, Garzanti, 1-141, 1989; Alice no País das Maravilhas, Mem Martins, Publicações Europa-America, 1998; Alisa în țara minunilor, Craiova, Editura Literatorul, 1991; Alice im Wunderland, Hamburg, Cecilie Dressler Verlag, 1990 ; Przygody Alicji w Krainie Czarów, Varsovie, Lettrex, 1990. (A)

$8^{\circ}$ Sienkiewicz, H. Quo Vadis, Varsovie, Labos, 1990; Quo vadis, Paris, Le livre de poche 3161, 1971; Quo vadis? Madrid, Aguilar, 1967; Quo vadis ?, Milan, Biblioteca 
Universale Rizzoli, 1984; Quo Vadis ?, Mem Martins, Livros de bolso Europa-America, 1974 ; Quo Vadis, Bucarest, Editura Universul, 1945; Quo Vadis?, Sawtry-New York, Hippocrene-Dedalus, 1993; Quo Vadis, Zurich, Diogenes, 1985 (chap. X-XII du premier volume). (Q)

$9^{\circ}$ Andrzejewski, J. Popiót $i$ diament, Varsovie, Krajowa Agencja Wydawnicza, 1966 ; Cendres et diamant, Paris, Gallimard, 1967; Cenizas y diamantes, Barcelone, Luis de Caralt, 1966; Cenere e diamanti, Lerici Editori, Milan, 1961; Cenuşă şi diamant, Bucarest, Editura pentru Literatură Universală, 1968; Ashes and Diamond, Eveston, Northwestern Univ. Press, 1991 ; Asche und Diamant, Frankfurt/Main, Suhrkamp Verlag, 1984 (chap. IV et V) (CD)

$10^{\circ}$ Maupassant, G. de. Bel ami, Livros de bolso Europa-América, Guimarães Editores, 1972. (BA)

\section{Bibliographie}

Aranda Ortiz, Antonio 1990. La expresión de la causatividad en español actual. Libros Pórtico, Zaragoza.

Comrie, Bernard 1976. The syntax of causative constructions : Cross-language similarities and divergencies. In: Shibatani (1976b, 261-312).

Coşeriu, Eugenio 1988. Der romanische Sprachtypus. Versuch einer neuen Typologisierung der romanischen Sprachen. In : Energeia und Ergon 1 : 207-24.

Disterheft, Dorothy 1980. The syntactic development of the infinitive in IndoEuropean. Slavica Publishers, Columbus, Ohio.

Duffley, Patrick 1992. The English infinitive. Longman, London.

Gawełko, Marek 2001. Essai de classification fonctionnelle des langues romanes. In: Romance Philology $55: 21-40$.

Gawełko, Marek 2004. Quelques particularités de l'infinitif substantivé en espagnol et en portugais. In: Bulletin Hispanique 2 :615-35.

Givón, Talmy - Phil Young 2002. Cooperation and interpersonal manipulation in the society of intimates. In : Shibatani (2002a, 23-56).

Gołạb, Zbigniew 1968. The grammar of Slavic causatives. In: Henry Kučera (ed.): Amaerican contributions to the Sixth International Congress of Slavists, vol. 1, 71-94. Mouton, Prague.

Heinisch, R. 1977. Vergleichende Untersuchungen zu slovakischen Präfixverben und ihren deutschen Entsprechungen. Leipzig (inédit).

Hernanz Carbó, María Luisa 2000. El infinitivo. In : Ignecio Bosque-Violeta Demonte (eds) : Gramática descriptiva de la lengua española, 2197-356. Espasa, Madrid.

Iliescu, Maria 1993. Le factitif roumain. In: Revue Roumaine de Linguistique 38 : $297-305$.

Iliescu, Maria 1995. La factitif dans les langues romanes. In : Romanistik in Geschichte und Gegenwart $2: 1-14$.

Marstrander, Leiv-Otto 2000. La proposition infinitive et le passif. In : Lene Schøsler (ed.) : Actes du colloque international, Institut d'Études Romanes, Université de Copenhague, 5-7 mars 1998, 205-211. Museum Tusculanum Press, Copenhagen. 
Moeschler, Jacques 2003. L'expression de la causalité en français. In : Cahiers de Linguistique Française $25: 11-42$.

Mori, Olga 1980. Frases infinitivas preposicionales en la zona significativa causal. Gunter Narr Verlag, Tübingen.

Pobożniak, Tadeusz 1979. Die Causativa in der Hindi-Sprache. In : Folia Orientalia 20 : $5-21$.

Reboul, Anne 2003. Causalité, force dynamique et ramifications temporelles. In: Cahiers de linguistique française $25: 43-69$.

Rémi-Giraud, Sylvianne 1988. Les grilles de Procuste, Description comparée de l'infinitif en français, grec ancien, allemand, anglais et arabe. In : Sylvianne Rémi-Giraud (ed.) : L'infinitif. Une approche comparative, 11-68. Presse universitaire de Lyon, Lyon.

Robustelli, Cecilia 1995. La costruzione di Fare con l'Infinito in Italiano moderno. In: Studi e Saggi linguistici 25 : 199-272.

Sandfeld, Kristian 1965. Syntaxe du français contemporain : L'infinitif. Librairie Droz, Genève. (1 $1^{\text {ère }}$ éd. 1943).

Shibatani, Masayoshi 1976a. The grammar of causative constructions : A conspectus. In : Shibatani (1976b, 1-40).

Shibatani, Masayoshi (ed.) 1976b. The grammar of causative constructions (Syntax and semantics, vol. 6). John Benjamins, Amsterdam \& Philadelphia.

Shibatani, Masayoshi (ed.) 2002a. The grammar of causation and interpersonal manipulation. Academic Press, New York.

Shibatani, Masayoshi 2002b. Introduction: Some basic issues in the grammar of causation. In : Shibatani (2002a, 1-22).

Shibatani, Masayoshi-Prashant Pardeshi 2002. The causative continuum. In : Shibatani (2002a, 85-126).

Simone, Raffaele-Donato Cerbasi 2001. Types and diachronic evolution of Romance causative constructions. In : Romanische Forschungen 113 :441-473.

Termińska, Kamila 1983. Składnia czasowników kauzatywnych we współczesnym języku polskim. Uniwersytet Śląski, Katowice.

Urbańczyk, Stanisław - Marian Kucała 1999. Encyklopedia języka polskiego, $3^{\text {e }}$ éd. Ossolineum, Wrocław.

Vanvolsem, Serge 1983. L'infinito sostantivato in italiano. Accademia della Crusca, Firenze.

Remarks on make + infinitive type causative constructions in Romance and some other languages

This paper establishes the productivity of causative constructions in eight languages, two of which (English and French) are highly analytical, one (Polish) highly synthetic, and five show a moderate tendency to analyticity. The paper then proceeds to a typological explanation of the differences observed. The general conclusion is that the productivity of causative constructions is proportionate to the degree of analyticity of the language: it is the strongest in French and the weakest in Polish. English is an exception in that causative constructions occur relatively rarely in it, due to the relative infrequency of the infinitive. Keywords: causative, productivity, explanation, synthetic, analytical 